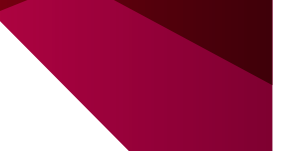

\title{
Co-existing proteinase 3-antineutrophil cytoplasmic antibody-associated vasculitis with immunoglobulin A nephropathy
}

\begin{abstract}
Adem Kucuk ${ }^{1}$, Yalcin Solak ${ }^{2}$, Abduzhappar Gaipov², Sinan Bagcaci ${ }^{3}$, Hasan Esen ${ }^{4}$, Suleyman Turk $^{2}$, and Recep Tunc ${ }^{1}$
\end{abstract}

Divisions of ${ }^{1}$ Rheumatology, ${ }^{2}$ Nephrology, ${ }^{3}$ Physical Medicine and Rehabilitation, and ${ }^{4}$ Pathology, Necmettin Erbakan University, Konya, Turkey
Received: June 3, 2014

Revised : October 12, 2014

Accepted: December 4, 2014

\section{Correspondence to}

Adem Kucuk, M.D.

Division of Rheumatology, Necmettin Erbakan University, 42090, Meram, Konya, Turkey

Tel: +90-332-223-6548

Fax: +90-332-223-6182

E-mail:drademk@yahoo.com
To the Editor,

Rapidly progressing immunoglobulin A (IgA) nephropathy usually presents with a histological pattern of focal or diffuse necrotizing crescentic glomerulonephritis. Antineutrophil cytoplasmic antibody (ANCA) and its specific antibody to antigen proteinase-3 (anti- $\mathrm{PR}_{3}$ ) are markers of pauci-immune crescentic nephritis, Wegener granulomatosis, and microscopic polyangiitis (PAN). The coexistence of ANCA positivity and IgA nephropathy has rarely been reported.

A 35-year-old man presented with arthralgia in the large joints, a palpable purpuric rash on both legs, swelling of the left knee (Fig. 1), difficulty swallowing, and epigastric discomfort for 2 weeks. His medical history was unremarkable. He was a nonsmoker and never used alcohol or illicit drugs. Three months earlier, he was evaluated for microscopic hematuria and was told that he had urinary stone disease. He was married and had two sons. He was afebrile and his blood pressure was $110 / 70 \mathrm{mmHg}$, pulse was regular at 80 beats per minute, and respiratory rate 18 breaths per minute. On physical examination, there was no erythema or thrush in the oral cavity. The abdomen was soft, and there was no organomegaly. There was tenderness in the epigastric region with voluntary guarding without rebound tenderness. He had palpable purpura on both legs and synovitis in the left knee joint without erythema.

The laboratory evaluation revealed the following: glucose (random) $125 \mathrm{mg} /$ $\mathrm{dL}$, creatinine $0.6 \mathrm{mg} / \mathrm{dL}$, urea $20 \mathrm{mg} /$
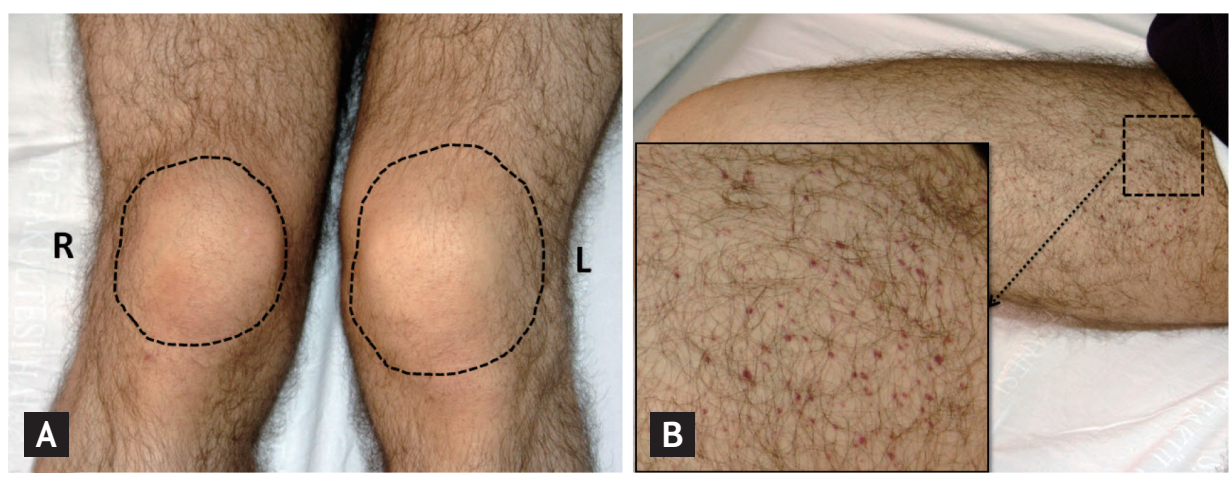

Figure 1. The patient's swollen left knee (A) and vasculitic lesions on the lower limb (B). 
dL, Na $138 \mathrm{mEq} / \mathrm{L}, \mathrm{K} 4.2 \mathrm{mEq} / \mathrm{L}$, lactate dehydrogenase $230 \mathrm{U} / \mathrm{L}$, total bilirubin $0.5 \mathrm{mg} / \mathrm{dL}$, alkaline phosphatase $48 \mathrm{U} / \mathrm{L}$, gamma glutamyl transferase $9 \mathrm{mg} / \mathrm{dL}$, alanine transaminase $24 \mathrm{U} / \mathrm{L}$, aspartate transaminase $25 \mathrm{U} / \mathrm{L}$, high-sensitive C-reactive protein $110 \mathrm{mg} / \mathrm{L}$, erythrocyte sedimentation rate $64 \mathrm{~mm} / \mathrm{hr}$, white blood cell $5.81 \times 10^{3} /$ $\mu \mathrm{L}$, eosinophils $0.120 \%$, hemoglobin $11.6 \mathrm{~g} / \mathrm{dL}$, platelets $187 \times 10^{3} / \mu \mathrm{L}$, international normalized ratio 1.11, activated partial thromboplastin time 27.7 , rheumatoid factor 23.2 $\mathrm{IU} / \mathrm{mL}$, complement component $31.38 \mathrm{~g} / \mathrm{L}$, complement component $40.225 \mathrm{~g} / \mathrm{L}$, and hepatitis serology negative for hepatitis $\mathrm{B}$ virus and hepatitis $\mathrm{C}$ virus. Immunologic markers were antinuclear antibody 1:80 (granular pattern), anti-dsDNA negative, and both IgG type cANCA and anti-PR3 positive. The Schirmer test was normal. Urinalysis showed microscopic hematuria, pyuria, and proteinuria. A 24-hour urine collection showed protein excretion of 3.135 g/day. Esophagogastroduodenoscopy revealed diffuse white plaques that were compatible with candidiasis. Anti-human immunodeficiency virus antibody was negative. Gastric mucosa biopsies showed gastritis with atrophy and intestinal metaplasia. Lymphoid aggregates were present but Helicobacter pylori was negative. We thought that the abdominal pain was due to microscopic PAN or Henoch-Schönlein purpura, and performed abdominal magnetic resonance angiography, which was normal. A punch biopsy of the skin of the leg with palpable purpura showed leukocytoclastic vasculitis without IgA deposition.

Paranasal and chest computed tomography did not reveal any pathological changes. Renal ultrasound showed normal-sized kidneys bilaterally, with normal echogenicity. A renal biopsy was compatible with IgA nephropathy (Fig. 2). There was no crescent formation or amyloid deposits.

The patient was administered oral prednisone $32 \mathrm{mg} \mathrm{a}$ day for presumptive vasculitis and fluconazole for esophageal candidiasis. After the ANCA antibody was found to be positive, we administered $1 \mathrm{~g}$ cyclophosphamide along with $500 \mathrm{mg}$ steroid pulses for 3 days. With this treatment, the palpable purpuric lesions disappeared and the 24-hour urinary protein excretion fell to $0.5 \mathrm{~g}$. After the steroid pulses, $1 \mathrm{mg} / \mathrm{kg}$ prednisone was given daily and the dosage was planned to be reduced by $25 \%$ at 2-week intervals. We planned to give the patient $1 \mathrm{~g}$ cyclophosphamide every month for 6 months. The patient
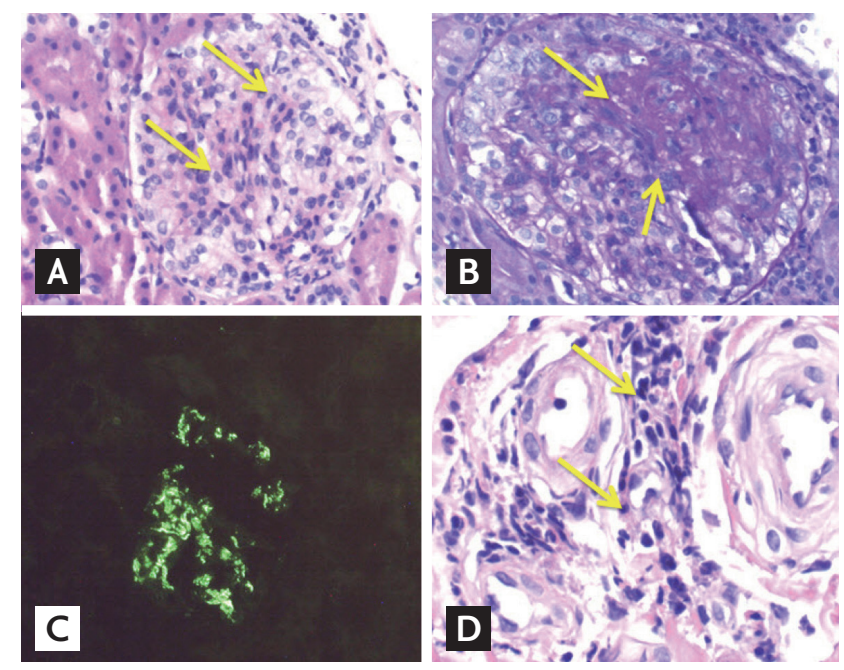

Figure 2. Light (A: H\&E, $\times 200$; B: PAS, $\times 200)$ and immunofluorescence (C) microscopy of the renal biopsy, and light microscopic images (D: H\&E, $\times 400$ ) of the skin biopsy. (A) and (B) show mesangial cellular proliferation (arrows), and increased mesangial matrix production, respectively. (C) depicts mesangial deposits of immunoglobulin A. (D) shows perivascular polymorphic neutrophil infiltration and extravasated erythrocytes (arrows).

responded well to this regimen without any systemic toxicity. The palpable purpura disappeared and proteinuria decreased to $0.5 \mathrm{~g}$ /day by 3 weeks after presentation. However, anti-PR3 was still positive.

The co-occurrence of IgA nephropathy with ANCA positivity has rarely been reported. Bantis et al. [1] retrospectively evaluated 393 patients with IgA nephropathy and found eight ANCA-positive patients (0.2\%), of whom only three were anti-PR3 positive. All of these patients had rapidly progressive glomerulonephritis with crescent formation in kidney biopsies. The anti-PR3-positive patients had pulmonary infiltrates or hemoptysis, proteinuria (in the nephrotic range in one and in the subnephrotic range in the other two patients), fever, and arthralgias. O'Donoghue et al. [2] screened 100 patients with IgA nephropathy and found two with ANCA positivity (2\%). Both patients were anti-myeloperoxidase positive and had no crescent formation in the kidney. Haas et al. [3] reported six patients with IgA nephropathy and ANCA positivity (five of them were anti-PR3 positive). All five anti-PR3 positive patients had impaired renal function, proteinuria (in the nephrotic range in two patients), hematuria, and hypertension. All of the patients had crescentic glomeruli in kidney biopsies, as well as sys- 
temic manifestations of vasculitic involvement.

Most of the anti-PR3-positive cases had severe kidney disease along with pulmonary or other manifestations of systemic vasculitis. However, our patient had only palpable purpura, subnephrotic proteinuria, and microscopic hematuria. He had no pulmonary involvement, creatinine elevation, crescent formation in the glomeruli, or hypertension. Our patient had IgG type ANCA. A punch biopsy of the skin revealed leukocytoclastic vasculitis without IgA deposition. In light of these clues, we diagnosed our patient with IgA nephropathy and ANCA associated vasculitis.

Generally, IgA nephropathy is considered a benign disease. In $30 \%$ of cases, renal function declines progressively, but slowly [4]. In most cases, the treatment is supportive. In a minority of cases, IgA nephropathy presents as rapidly progressive glomerulonephritis and these patients benefit from therapy that is analogous to the treatment administered in ANCA-associated vasculitis (steroids and cyclophosphamide) [5]. Patients with IgA nephropathy associated with ANCA antibodies should be treated in the same manner. Despite a lack of severe systemic manifestations of vasculitic disease, we administered one dose of cyclophosphamide to our patient along with pulse steroids and then oral corticosteroids.

In conclusion, we presented a rare case of vasculitic IgA nephropathy associated with anti-PR3 and ANCA positivity. Unlike other reported cases, our patient presented with mild renal and systemic involvement and showed a favorable response to cytotoxic treatment and corticosteroids.

Keywords: Anti-proteinase 3; Glomerulonephritis, IGA; Antibodies, antineutrophil cytoplasmic

\section{Conflict of interest}

No potential conflict of interest relevant to this article was reported.

\section{REFERENCES}

1. Bantis C, Stangou M, Schlaugat C, et al. Is presence of ANCA in crescentic IgA nephropathy a coincidence or novel clinical entity? A case series. Am J Kidney Dis 2010;55:259-268.

2. O'Donoghue DJ, Nusbaum P, Noel LH, Halbwachs-Mecarelli L, Lesavre P. Antineutrophil cytoplasmic antibodies in IgA nephropathy and Henoch-Schonlein purpura. Nephrol Dial Transplant 1992;7:534-538.

3. Haas M, Jafri J, Bartosh SM, Karp SL, Adler SG, Meehan SM. ANCA-associated crescentic glomerulonephritis with mesangial IgA deposits. Am J Kidney Dis 2000;36:709-718.

4. Floege J, Eitner F. Current therapy for IgA nephropathy. J Am Soc Nephrol 2011;22:1785-1794.

5. Harper L, Ferreira MA, Howie AJ, et al. Treatment of vasculitic IgA nephropathy. J Nephrol 2000;13:360-366. 